\title{
FINITE GROUP WITH HALL COVERINGS
}

\author{
ENRICO JABARA and MARIA SILVIA LUCIDO
}

(Received 1 November 2001; revised 11 June 2003)

Communicated by R. Howlett and E. A. O'Brien

\begin{abstract}
In this paper we describe the groups admitting a covering with Hall subgroups. We also determine the groups with a $\pi_{1}-$ Hall subgroup, where $\pi_{1}$ is the connected component of the prime graph, containing the prime 2.
\end{abstract}

2000 Mathematics subject classification: primary 20D20, $20 \mathrm{D} 25$.

\section{Introduction}

In this paper we study the Hall coverings, defined as follows. A Hall covering of a finite group $G$ is a set $\mathscr{H}=\left\{H_{1}, H_{2}, \ldots, H_{r}\right\}$ of proper Hall subgroups of $G$ such that:

(a) $\bigcup_{i=1}^{r} H_{i}=G$ and

(b) either $\left|H_{i}\right|=\left|H_{j}\right|$ or $\left(\left|H_{i}\right|,\left|H_{j}\right|\right)=1$ for $i, j=1, \ldots, r$.

If the elements of $\mathscr{H}$ all have order a prime power, then $\mathscr{H}$ is called a Sylow covering of $G$. The finite groups $G$ with a Sylow covering have been studied independently by Higman [10] and Zacher [27, 28] in the case in which $G$ is soluble, by Suzuki [25] in the case of a simple group $G$ and by Brandl [2] in the general situation. This last paper has a missing case, which we consider here.

We want to study the groups which admit a Hall covering. It is clear that if a group $G$ admits a Hall covering, then its prime graph is not connected. We shall also see that if $G$ admits a Hall covering, than $G$ has a $\pi_{1}$-Hall subgroup, where $\pi_{1}$ is the connected component of the prime graph of $G$ containing the prime 2 .

(C) 2005 Australian Mathematical Society $1446-7887 / 05 \$ A 2.00+0.00$ 
It is well known that $G$ is a soluble group if and only if $G$ has a $\pi$-Hall subgroup for any set of primes $\pi$. If $G$ is not soluble, the existence of some Hall subgroups have been proved in several papers (see, for example, $[9,23,8]$ ). We prove the following theorem on the existence of a $\pi_{1}$-Hall subgroup. We suppose that the group $G$ is not soluble and that $G$ is not a Frobenius group. In fact if $G$ is a non-soluble Frobenius group, the Frobenius complement is isomorphic to a direct product of $S L(2,5)$ with a $\{2,3,5\}^{\prime}$-group with cyclic Sylow subgroups. The Frobenius complements are $\pi_{1}$-Hall subgroups and they are all conjugate (see [12, page 387]).

THEOREM A. Let $G$ be a non-soluble group in which the prime graph is not connected. Suppose further that $G$ is not a Frobenius group. Then $G$ has a $\pi_{1}$-Hall subgroup if and only if $G / \operatorname{Fit}(G)$ is one of the groups in Table 1 .

We also classify the groups which admit a $\pi$-Hall subgroup for any connected subset $\pi$ of $\pi(G)$ (see Corollary 3.6).

We prove the following theorem, describing the finite groups admitting a Hall covering.

THEOREM B. Let $G$ be a group in which the prime graph is not connected. Then $G$ admits $a$ Hall covering if and only if either

(i) $G$ is a Frobenius or a 2-Frobenius group or

(ii) $G / \operatorname{Fit}(G)$ is isomorphic to one of the following groups: $\operatorname{PSL}(2, q), \operatorname{PSL}(3,4)$, $\operatorname{PSL}(3, q)$ with $(3, q-1)=1, S z(q), A_{7}, M_{22}, M(q)$.

Another class of groups related to groups admitting Hall coverings is the class of groups with a partition (see [22, Section 3.5]) and the $C N$-groups, that is groups in which the centralizer of any non trivial element is nilpotent (see [7, Chapter 10]). We shall see how these groups are strictly related to nilpotent Hall coverings.

The results of this paper depend upon the classification.

\section{Notation and preliminary results}

All the groups considered in this paper are finite. If $G$ is a group we denote by $\pi(G)$ the set of prime divisors of $|G|$. If $\mathscr{H}$ is a Hall covering of the group $G$, we define $\pi(\mathscr{H})=\left\{\pi\left(H_{i}\right) \mid i=1,2, \ldots, r\right\}$; then $\pi(\mathscr{H})=\left\{\sigma_{1}, \sigma_{2}, \ldots, \sigma_{s}\right\}$ with $\sigma_{i} \cap \sigma_{j}=\emptyset$ if $i \neq j$ (and obviously $s<r$ ). We suppose that if $i<j$, then $\min \sigma_{i}<\min \sigma_{j}$ (in particular if $2 \in \pi(G)$ then $\left.2 \in \sigma_{1}\right)$.

If $G$ is a group, we define its prime graph $\Gamma(G)=\Gamma$ as follows: the set of vertices of $\Gamma$ is $\pi(G)$ and two vertices $p$ and $q$ are connected $(p \sim q)$ if and only if there exists in $G$ an element of order $p q$. Let $\pi_{1}, \pi_{2}, \ldots, \pi_{t}$ be the connected components of $\Gamma$ 
and let $t(G)=t$ be the number of such connected components; we suppose $2 \in \pi_{1}$, if $2 \in \pi(G)$. Then $\pi(G)$ is the disjoint union of the $\pi_{i}, i=1,2, \ldots, t$. Moreover, if $G$ admits a Hall covering $\mathscr{H}$, then any element of $\pi(\mathscr{H})$ is a disjoint union of certain connected components of $\Gamma(G)$, in particular, $2 \leq s \leq t$.

If $\mathscr{C}_{1}$ and $\mathscr{C}_{2}$ are two classes of groups, a group $G$ is $\mathscr{C}_{1}$-by- $\mathscr{C}_{2}$ if $G$ has a normal subgroup $N$ with $N \in \mathscr{C}_{1}$ and $G / N \in \mathscr{C}_{2}$.

We denote by $\operatorname{Fit}(G)$ the Fitting subgroup of $G$, that is, the maximal normal nilpotent subgroup of $G$.

A group $G$ is an almost simple group if there exists a simple non-abelian group $S$ such that $S \leq G \leq \operatorname{Aut}(S)$.

Let $p$ be an odd prime and $q=p^{2 f}$. We denote by $M(q)$ the non split extension of $\operatorname{PSL}(2, q)$, with $|M(q): P S L(2, q)|=2$.

A proper subgroup $H$ of $G$ is isolated (in $G$ ) if

(a) $H \cap H^{g}=1$ for any $g \notin N_{G}(H)$;

(b) $C_{G}(h) \leq H$ for any $1 \neq h \in H$.

The notation for the simple groups follows the one of [5]. For the rest, the notation will be standard (see, for example, [7] and [12]).

A group is called 2-Frobenius if it has two normal subgroups $N, K$, with $N<K$, such that $K$ and $G / N$ are Frobenius groups.

The following results were proved in an unpublished paper of Gruenberg and Kegel, but they can be found in [26]

PROPOSITION 2.1 ([26]). If $G$ is a group whose prime graph has more than one connected component, then $G$ has one of the following structures:

(a) $G$ is a Frobenius or a 2-Frobenius group.

(b) $G$ is simple.

(c) $G$ is simple by $\pi_{1}$.

(d) $G$ is $\pi_{1}$ by simple by $\pi_{1}$.

Moreover, if $G$ is not soluble and $\pi_{i}$ is a component of $\Gamma(G)$ with $i>1$, then $G$ has an isolated $\pi_{i}$-Hall subgroup.

COROLlARY 2.2. If $G$ is a soluble group with a Hall covering, then $|\pi(\mathscr{H})|=2=$ $t(G)$ and $G$ is a Frobenius or a 2-Frobenius group.

It is well known that $G$ is soluble if and only if $G$ has a $\pi$-Hall subgroup for any $\pi \subseteq \pi(G)$; moreover any two of them are conjugate. If we want to consider the general case, we first have to deal with the existence of $\pi_{i}(G)$-Hall subgroups of $G$. We recall the following:

Proposition 2.3 ([8]). If $G$ has a $\pi$-Hall subgroup with $2 \notin \pi$, then the $\pi$-Hall subgroups are all conjugate. 
Therefore by Proposition 2.1 and Proposition 2.3, we know that if $t(G) \geq 2$, then there exists a $\pi_{i}$-Hall subgroup for any $i \geq 2$ and these are all conjugate.

We want to examine now the groups which admit a $\pi_{1}$-Hall subgroup.

\section{3. $\pi_{1}$-Hall subgroups}

By the preceding remarks, we can assume that

(*) $\quad G$ is a non-soluble group in which the prime graph is not connected and $G$ is not a Frobenius group.

The aim of this section is to prove the following:

PROPOSITION 3.1. Let $G$ be a group satisfying $(*)$. Then $G$ has a $\pi_{1}$-Hall subgroup if and only if $G / \mathrm{Fit}(G)$ is one of the groups of Table 1.

We begin with some general remarks.

LEMMA 3.2. Let $G$ be a group satisfying (*).

(i) If $R$ is the maximal normal soluble subgroup of $G$, then $R=\operatorname{Fit}(G)=O_{\pi_{1}}(G)$ and $G / \operatorname{Fit}(G)$ is isomorphic to an almost simple group. Moreover if $S$ is the only simple non-abelian section of $G$, we have $\pi_{i}(G)=\pi_{i}(S)$, for $i \geq 2$.

(ii) $G$ has a $\pi_{1}$-Hall subgroup if and only if $G / \operatorname{Fit}(G)$ has $a \pi_{1}$-Hall subgroup.

Proof. (i) It can be easily deduced by the results in the paper [26].

(ii) Let $F=\operatorname{Fit}(G)$. If $\bar{G}=G / F$ has a $\pi_{1}(\bar{G})$-Hall subgroup $\bar{H}$, then of course $H$ is a $\pi_{1}(G)$-Hall subgroup of $G$, since $\pi(H) \subseteq \pi_{1}(G)$.

Let now $H$ be a $\pi_{1}(G)$-Hall subgroup of $G$, then $F \leq H$. Otherwise $F H>H$ and $F H$ is also a $\pi_{1}(G)$-subgroup of $G$, contradicting the maximality of $H$. Therefore $H / F$ is a $\pi_{1}(G / F)$-Hall subgroup of $G / F$.

The aim of the following sections is therefore to prove:

PROPOSITION 3.3. If $G$ is an almost simple group, then $G$ has a $\pi_{1}$-Hall subgroup if and only if $G$ is one of the groups of Table 1.

In Tables 1 and 2, we suppose that $r$ is an odd prime number, $p$ is a prime number, $q=p^{f}$ and $P$ is a Sylow $p$-subgroup of $G$. We use the notation of the Atlas [5].

We denote by $H$ a $\pi_{1}(G)$-Hall subgroup of $G$ and we write in the third column the structure of a representative of the conjugacy classes of the $\pi_{1}(G)$-Hall subgroups of $G$. In the last column we write some remarks concerning $H$. We also recall that $A_{5} \cong \operatorname{PSL}(2,4) \cong \operatorname{PSL}(2,5)$ and $A_{6} \cong \operatorname{PSL}(2,9)$. 
If $G$ is $\operatorname{PSL}(n, q)$, we denote by $P_{\mathrm{I}^{\prime}}, P_{n^{\prime}}$ the maximal parabolic subgroups of type $P_{J}$, with $J$ respectively $\Pi \backslash\{1\}$ and $\Pi \backslash\{n\}$, as described in Remark 1 in Section 4 .

We observe that the following groups admit also a $\pi$-Hall subgroup, with $\pi$ a set of primes strictly containing $\pi_{1}$.

3.1. Simple groups If $G$ admits a Hall covering, then the number $t(G)$ of connected components of the prime graph $\Gamma(G)$ is greater than or equal to 2 . We first suppose $t(G)=2$, and therefore $\pi_{i}=\sigma_{i}$ for $i=1,2$.

We recall that a group is said to be factorizable by two proper subgroups $A$ and $B$ if $G=A B=B A$.

LEMMA 3.4. Let $G$ be a finite group with $t(G)=2$. If $G$ has a $\pi_{1}$-Hall subgroup $A$, then $G$ is factorizable by $A$ and another proper subgroup $B$ such that $(|A|,|B|)=1$.

Proof. If $G$ has a $\pi_{1}$-Hall subgroup $A$, then by Proposition $2.1, G$ has also a $\pi_{2}-\mathrm{Hall}$ subgroup $B$. Then $(|A|,|B|)=1$ and $|G|=|A||B|$, and therefore $G=A B$.

Let now $G$ be a simple group. Then by Lemma 3.4, $G$ is factorizable by two proper subgroups $A$ and $B$ and we can assume $A$ to be a $\pi_{1}$-Hall subgroup and $B$ a $\pi_{2}$-Hall subgroup. We can therefore conclude by [1, Theorem 1.1] that $G$ is one of the following:

(i) $A_{r}$, with $r \geq 5$ a prime and $r-2$ not a prime, then $A \cong A_{r-1}$;

(ii) $\operatorname{PSL}(r, q)$, with $r$ an odd prime such that $(r, q-1)=1$ and either $G \cong$ $\operatorname{PSL}(5,2)$ and $|B|=5.31$ or $A$ is a maximal parabolic subgroup such that $P S L(r-1, q)$ is involved in $A$.

We observe that in the case $\operatorname{PSL}(5,2)$ with $|B|=5 \cdot 31, A$ is not a $\pi_{1}$-Hall subgroup because $5 \in \pi_{1}$.

We now suppose that $G$ is a simple non-abelian group with $t(G) \geq 3$. We consider separately the case in which $G$ is a sporadic or an alternating group and the case in which $G$ is a simple group of Lie type. In the following we look for $\pi$-Hall subgroups of $G$, with $\pi$ a set of primes in $\pi(G)$ containing $\pi_{1}$. We use the results in [26], without further reference.

Alternating groups Since $A_{5} \simeq \operatorname{PSL}(2,5)$ and $A_{6} \simeq \operatorname{PSL}(2,9)$, it is enough to consider $A$, with $r \geq 7, r$ and $r-2$ primes. The maximal subgroups of the alternating groups have been classified (see for example [6, Theorem 5.2A]). The only cases in which $A_{r}$ ( $r \geq 2, r$ and $r-2$ primes) admits a $\{r-2, r\}^{\prime}$-Hall subgroup $H$ is for $r=7$. In fact, by point (i) of [6, Theorem 5.2A], we should have

$$
H \leq\left(A_{r-3} \times A_{3}\right)\langle x\rangle, \quad \text { with } x \text { of order } 2
$$

but if $r>7$, then $\left(A_{r-3} \times A_{3}\right)\langle x\rangle$ has index greater than $r(r-2)$ in $A_{r}$. 
TABLE 1.

\begin{tabular}{|c|c|c|c|}
\hline$G$ & Conditions & $H$ & Remarks \\
\hline$A_{7}$ & & $\left(A_{4} \times A_{3}\right) .2$ & soluble \\
\hline$\overline{A_{r}}$ & $r-2$ not a prime & $A_{r-1}$ & simple \\
\hline$M_{11}$ & & $3^{2}: Q_{8} .2$ & soluble \\
\hline$M_{22}$ & & $2^{4}: A_{6}$ & \\
\hline$M_{23}$ & & $\begin{array}{c}P S L(3,4): 2_{2} \\
2^{4}: A_{7}\end{array}$ & \\
\hline$J_{1}$ & & $2 \times A_{5}$ & \\
\hline $\operatorname{PSL}(2, q)$ & $q=2^{n}$ & $P$ & nilpotent \\
\hline $\operatorname{PSL}(2, q)$ & $\begin{array}{c}q \equiv 1(4) \\
q \neq 13,25,61\end{array}$ & $D_{q-1}$ & soluble \\
\hline $\operatorname{PSL}(2, q)$ & $\begin{array}{c}q \equiv-1(4) \\
q \neq 11,23,59\end{array}$ & $D_{q+1}$ & soluble \\
\hline $\operatorname{PSL}(2, q)$ & $q=11,13$ & $\begin{array}{l}D_{12} \\
A_{4} \\
\end{array}$ & $\begin{array}{l}\text { soluble } \\
\text { soluble }\end{array}$ \\
\hline $\operatorname{PSL}(2, q)$ & $q=23,25$ & $\begin{array}{c}D_{24} \\
S_{4}, 2 \text { classes } \\
\end{array}$ & $\begin{array}{l}\text { soluble } \\
\text { soluble }\end{array}$ \\
\hline $\operatorname{PSL}(2, q)$ & $q=59,61$ & $\begin{array}{c}D_{60} \\
A_{5}, 2 \text { classes } \\
\end{array}$ & $\begin{array}{l}\text { soluble } \\
\text { simple }\end{array}$ \\
\hline $\operatorname{PSL}(3, q)$ & $q=2^{2}$ & $P$ & nilpotent \\
\hline $\operatorname{PSL}(r, q)$ & $(r, q-1)=1$ & $\begin{array}{l}P_{1^{\prime}} \\
P_{r^{\prime}}\end{array}$ & \\
\hline$S z(q)$ & $q=2^{f}, f$ odd & $P$ & nilpotent \\
\hline$S_{7}$ & & $S_{6}$ & almost simple \\
\hline$S_{r}$ & $r-2$ not a prime & $S_{r-1}$ & almost simple \\
\hline $\begin{array}{c}P S L(2, q)\langle\alpha\rangle \\
q=2^{n}\end{array}$ & $\begin{array}{l}\alpha \text { field automorphism } \\
\qquad|\alpha|=2^{m}\end{array}$ & $N_{G}(P)$ & soluble \\
\hline$M(q)$ & & $D_{2(q-1)}$ & soluble \\
\hline $\begin{array}{c}P S L(r, q)\langle\alpha\rangle \\
(r, q-1)=1\end{array}$ & $\begin{array}{l}\alpha \text { field automorphism } \\
\qquad|\alpha|=r^{m}\end{array}$ & $\begin{array}{l}P_{1^{\prime}}\langle\alpha\rangle \\
P_{r^{\prime}}\langle\alpha\rangle\end{array}$ & \\
\hline
\end{tabular}

TABLE 2.

\begin{tabular}{|c|c|c|c|}
\hline$G$ & $\pi$ & $\pi$-Hall subgroup & Remarks \\
\hline$A_{7}$ & $\pi_{1} \cup\{5\}$ & $A_{6}$ & simple \\
\hline$M_{11}$ & $\pi_{1} \cup\{5\}$ & $M_{10}=M(9)$ & almost simple \\
\hline$M_{23}$ & $\pi_{1} \cup\{11\}$ & $M_{22}$ & simple \\
\hline$P S L(3, q), q=2^{2}$ & $\{2,3\}$ & $N_{G}(P)$ & soluble \\
\hline$P S L(2, q), q=2^{n}$ & $\pi(q(q-1))$ & $N_{G}(P)$ & soluble \\
\hline$P S L(2,7)$ & $\pi\left(q^{2}-1\right)$ & $S_{4}$ & soluble \\
\hline$P S L(2,11)$ & $\pi\left(q^{2}-1\right)$ & $A_{5}, 2$ classes & simple \\
\hline$S z(q)$ & $\pi(q(q-1))$ & $N_{G}(P)$ & soluble \\
\hline
\end{tabular}


Sporadic groups Let $G$ be a sporadic group and $h=|G| /|G|_{\pi_{1}}$. If $G$ has a $\pi$-Hall subgroup, with $\pi_{1} \subseteq \pi \subset \pi(G)$, then it must have a maximal subgroup of order dividing $h$. Then using the Tables in [26] and the Atlas [5], it is easy to check that the following groups do not have maximal subgroups dividing $h: M_{24}, J_{3}, J_{4}, H S, S u z$, $O^{\prime} N, L y, \mathrm{Co}_{2}, F_{23}, T h$. For the other sporadic groups we use the following arguments.

Let $\chi_{2}$ be the non principal character of minimal degree of $F_{24}^{\prime}$. Then $\operatorname{deg}\left(\chi_{2}\right)=$ 8671 . Since $17 \cdot 23 \cdot 29=11339$ (and any other character of $F_{24}^{\prime}$ has greater degree), then $F_{24}^{\prime}$ hasn't subgroups whose index divides $17 \cdot 23 \cdot 29$.

Let $\chi_{2}$ be the non principal character of minimal degree of $M$. Then $\operatorname{deg}\left(\chi_{2}\right)>$ $41 \cdot 59 \cdot 71$ and therefore $M$ hasn't subgroups whose index divides $41 \cdot 59 \cdot 71$.

Let $\chi_{2}$ be the non principal character of minimal degree of $B M$. Then $\operatorname{deg}\left(\chi_{2}\right)>$ 31.47 and therefore $B M$ hasn't subgroups whose index divides $31 \cdot 47$.

Simple groups of Lie type We now consider a finite simple group of Lie type defined over a field with $q=p^{f}$ elements. We recall that a Singer cycle of $\operatorname{PSL}(n, q)$ is an element of order $\left(q^{n}-1\right) /(q-1)(n, q-1)$.

If $G$ is a simple group of Lie type with $t(G) \geq 3$, then $G$ is one of the following (see $[13,14,26]): \operatorname{PSL}(2, q), \operatorname{PSL}(3,4), E_{7}(2), E_{7}(3), E_{8}(q), F_{4}(q)$ with $q$ even, $G_{2}(q)$ with $q \equiv 0(3), \operatorname{PSU}(6,2), S z(q),{ }^{2} D_{p}(3)$ with $p=2^{n}+1, n \geq 2,{ }^{2} E_{6}(2)$, ${ }^{2} F_{4}(q), \operatorname{Ree}(q)$.

We first observe that if $G$ is $\operatorname{PSL}\left(2,2^{n}\right), \operatorname{PSL}(3,4)$ or $S z(q)$, then $\pi_{1}(G)=\{2\}$. Therefore a $\pi_{1}$-Hall subgroup is in fact a Sylow 2-subgroup. Also for $\operatorname{PSL}(2, q), q$ odd, it is easy to see that a $\pi_{1}$-Hall subgroup exists and they are all conjugate.

We begin with an easy remark, which allows us to understand the structure of the maximal parabolic subgroups of a finite group of Lie type.

REMARK 1. Let $J$ be a subset of the set $\Pi$ of fundamental roots of the finite group of Lie type $G$ and $\Phi_{J}$ be the set of roots which are integral combinations of roots in $J$. Let $L_{J}$ be the subgroup of $G$ generated by $H$ and the root subgroups $X_{r}$, for all $r \in \Phi_{J}$. Then $P_{J}=U_{J} L_{J}, L_{J} \cap U_{J}=1$ and $U_{J}$ is an unipotent subgroup, by [4, Theorem 8.5.2]. If $P_{J}$ is a maximal parabolic subgroup, then $J=\Pi \backslash\{i\}$, for some fundamental root $i$. Since $H$ normalises any $X_{r}$, we have $L_{J}=\left\langle X_{r}: r \in \Phi_{J}\right\rangle H_{i}$, where $H_{i}$ is the subgroup of $G$ generated by $h_{i}(\lambda), \lambda \in K^{*}$ (see [4, page 98]). We call $M_{J}=\left\langle X_{r}: r \in \Phi_{J}\right\rangle$.

We begin with a case by case analysis.

Let $G$ be one of the groups listed in Table 3. We suppose that there exists $K$ a $\pi$-Hall subgroup of $G$, with $\pi_{1} \subseteq \pi$. We want to prove that $K$ cannot be contained in any maximal subgroup of $G$, and therefore $G$ does not admit any $\pi_{1}$-Hall subgroup. We use the Theorem of [17], observing that $|K| \geq q^{k(G)}$, where $q^{k(G)}$ is as defined in [17, Table 1], and also in our Table 2. If $M$ is a maximal subgroup of $G$ containing $K$, 
TABLE 3.

\begin{tabular}{|c|c|c|}
\hline$G$ & $q^{k(G)}$ & $h(G)$ \\
\hline$E_{7}(q)$ & $q^{64}$ & $q^{7}+1$ \\
$E_{8}(q)$ & $q^{110}$ & $\left(q^{12}-1\right)^{2}$ \\
$F_{4}(q)$ & $q^{24}$ & $\left(q^{6}-1\right)^{2}$ \\
$G_{2}(q)$ & $q^{6}$ & $\left(q^{2}-1\right)^{2}$ \\
${ }^{2} E_{6}(q)$ & $q^{37}$ & $\left(q^{6}-1\right)^{2}$ \\
\hline
\end{tabular}

then

(1) $M$ contains a $p$-Sylow subgroup of $G\left(q=p^{f}\right)$,

(2) $|M| \geq q^{k(G)}$,

(3) $|M|$ is divisible by $h(G)$, where $h(G)$ is an integer, as listed in Table 2.

By [17, Theorem], $M$ is either a parabolic subgroup or $M$ is as in [17, Table 1]. The groups listed in [17, Table 1] do not contain a $p$-Sylow subgroup of $G$. Moreover, if we consider the maximal parabolic subgroups of $G$, we can easily check that no one of them has order divisible by $h(G)$. We conclude that $G$ does not admit a $\pi$-Hall subgroup.

$\operatorname{PSU}(6,2)$ It can be checked in the Atlas [5] that there is no $\pi$-Hall subgroup, for $\pi_{1} \subseteq \pi$.

${ }^{2} D_{n}$ (3) It can be proved that if $K$ is a maximal subgroup containing a 3-Sylow subgroup, then $K$ is a parabolic subgroup (by [16] and some easy calculations). If we denote by $i$ the $i$ th node in the Dynkin diagram, then the isomorphism classes of the maximal parabolic subgroups are

$$
\begin{aligned}
& J=\Pi \backslash\{i\} \quad \Rightarrow \quad M_{J} \cong A_{i-1}(q) \times{ }^{2} D_{n-i}(q), \text { for } 1 \leq i \leq n-4, \\
& J=\Pi \backslash\{n-3\} \quad \Rightarrow \quad M_{J} \cong A_{n-4}(q) \times{ }^{2} A_{3}(q), \\
& J=\Pi \backslash\{n-2\} \quad \Rightarrow \quad M_{J} \cong A_{n-3}(q) \times A_{1}\left(q^{2}\right) \\
& J=\Pi \backslash\{n-1\} \quad \Rightarrow \quad M_{J} \cong A_{n-2}(q) .
\end{aligned}
$$

Since the $p^{\prime}$-part of the order of the maximal parabolic subgroup $P_{J}$ is $(q-1)\left|M_{J}\right|$, it can be easily seen that $q^{n-1}-1$ does not divide the order of any maximal parabolic subgroup of $G$, while $q^{n-1}-1$ should divide the order of a $\pi$-Hall subgroup of $G$, $\pi_{1} \subseteq \pi$.

${ }^{2} F_{4}(q)$ We know from [19], that the only maximal subgroups containing a $p$-Sylow subgroup of $G$ are the maximal parabolic subgroups, and no one of these is divisible by $q^{6}+1$, which should divide the order of a $\pi$-Hall subgroup of $G, \pi_{1} \subseteq \pi$.

${ }^{2} G_{2}(q)$ We know from [15], that the only maximal subgroups containing a $p$-Sylow subgroup of $G$ are the maximal parabolic subgroups, and no one of these is divisible by $q^{2}+1$, which should divide the order of a $\pi$-Hall subgroup of $G, \pi_{1} \subseteq \pi$. 
3.2. Almost simple groups The connected components of the prime graph of almost simple groups have been calculated in [18]. We therefore refer to [18], without further reference.

For the sporadic groups we refer again to [5]. For the alternating groups, it is easy to observe that if $G=S_{r}$ is the symmetric group over $r$ elements, with $r$ an odd prime, $r \geq 7$, then the stabiliser of an element is isomorphic to $S_{r-1}$ and it is a $\pi_{1}$-Hall subgroup. Moreover the $\pi_{1}$-Hall subgroups are all conjugate.

If $S \cong P S L(2, q)$ and $G$ contains a diagonal automorphism, then $\pi\left(q^{2}-1\right) \subseteq \pi_{1}(G)$ and $P G L(2, q)$ does not contain subgroups of order (divisible by) $q^{2}-1$.

If $G$ contains a field automorphism $\alpha$ of order not a power of 2 and $q \neq 2$ or 3 , then $\Gamma(G)$ is connected. If $|\alpha|=2$ and $G=S\langle\alpha\rangle$, then $\pi_{1}(G)=\pi(q(q-1))$. If $q$ is odd, then there is no $\pi_{1}(G)$-Hall subgroup in $G$, since there isn't a $\pi_{1}(G)$-Hall subgroup in $S$. If $q$ is even, let $B$ be the subgroup of $S$ of the upper triangular matrices. We observe that $B$ is fixed by $\alpha$ and therefore $\widetilde{B}=B\langle\alpha\rangle$ is a $\pi_{1}$-Hall subgroup of $G$. Moreover the $\pi_{1}$-Hall subgroups of $G$ are all conjugate.

If $f$ is an odd prime and $q=2^{f}$ or $q=3^{f}$, then $\pi_{1}(G)=\pi(f q(q+1) /(2, q-1))$. If $K$ is a $\pi_{1}(G)$-Hall subgroup of $G$, then $K \cap S$ is a subgroup of $S$ of order $q(q+1) /(2, q+1)$, which does not exist.

If $q$ is odd and a square, that is $q=q_{0}^{2}$, for some $q_{0}=p^{n}$, then there exists a non-split extension $M(q)$ of $\operatorname{PSL}(2, q)$ of order 2 , with $\Gamma(M(q))=\Gamma(S)$. We observe that the order of a $\pi_{1}$-Hall subgroup of $G$ should be $2(q-1)$ and therefore a $\pi_{1}$-Hall subgroup of $S$ is $N_{S}(H)=N$ the normaliser of the diagonal group $H$. We also observe that $H$, and therefore $N$, is fixed by any automorphism of $S$. Then $G$ has a $\pi_{1}$-Hall subgroup.

If $S=S z(q)$ with $q=2^{f}$, and $G$ is a subgroup of its automorphism group, then $\Gamma(G)$ is always connected, except when $f$ is a prime and $G=S\langle\alpha\rangle$, with $\alpha$ a field automorphism of order $f$. In this case $\pi_{1}(G)=\pi(2 f(q+\sqrt{2 q}+1))$ or $\pi_{1}(G)=\pi(2 f(q-\sqrt{2 q}+1))$ depending if $f \equiv 1,7$ (8) or $f \equiv 3,5(8)$. In both cases there should exists a $\pi(2(q \pm \sqrt{2 q}+1)$-Hall subgroup of $S$ and this is not possible in any of the two cases (see [12] or [25]).

If $S \cong P S L(3,4)$, it is easy to check (see [5]) that there is no $\pi_{1}$-Hall subgroup for any of the extensions.

If $S \cong P S L(r, q)$ with $(r, q-1)=1$ and $q=p^{f}, p$ a prime, then $\operatorname{Aut}(S)=$ $S(\langle\varphi, \tau\rangle)$, where $\varphi$ is a field automorphism of order $f$, and $\tau$ is the graph automorphism of order 2 of $S$.

If $G$ contains a graph automorphism and $t(G)=2$, then there is no $\pi_{1}(G)$-Hall subgroup in $G$. In fact, no $\pi_{1}(S)$-Hall subgroup of $G$ is fixed by $\alpha$, which interchanges the two conjugacy classes of parabolic subgroups.

If $G$ contains a field automorphism of order a prime different from $r$, then $\Gamma(G)$ is connected. If $G=S\langle\alpha\rangle$ with $\alpha$ a field automorphism of order $r$, then $\pi_{1}(G)=\pi_{1}(S)$ 
and $C_{S}(\alpha) \cong P G L\left(3, q_{0}\right)$ if $q_{0}^{3}=q$. We observe that there exists a $\pi_{1}$-Hall subgroup $\widetilde{P}_{1}$ of $G$, which is an extension of $P_{1}$, a $\pi_{1}$-Hall subgroup of $S$.

By the proof of Proposition 3.1 and Proposition 3.3, we also get the following corollaries.

COROLLARY 3.5. Let $G$ be a group and $\pi$ be a set of primes such that $\pi_{1} \subseteq \pi \subset$ $\pi(G)$. Then

(i) $G$ has a $\pi$-Hall subgroup if and only if $G$ has a $\pi_{1}$-Hall subgroup;

(ii) if $\pi_{1} \subset \pi$ and $G$ satisfies (*), then $G / \operatorname{Fit}(G)$ is isomorphic to one of the groups in Table 2.

Let $G$ be a group and $\pi$ be a set of primes in $\pi(G)$. We say that $\pi$ is connected if and only if there exists $i=1, \ldots, t(G)$ such that $\pi \subseteq \pi_{i}$.

COROLLARY 3.6. Let $G$ be a group satisfying (*). Then $G$ has a $\pi$-Hall subgroup, for any connected subset $\pi$ of $\pi(G)$ if and only if $G / \mathrm{Fit}(G)$ is isomorphic to one of the following groups: $\operatorname{PSL}(2, q), \operatorname{Sz}(q), \operatorname{PSL}(3,3), \operatorname{PSL}(3,4), A_{7}, M_{11}, \operatorname{PSL}\left(2,2^{n}\right)\langle\alpha\rangle$ with $|\alpha|=2^{m}, M(q)$.

PROOF. It is enough to examine the non-soluble groups $H$ in Table 1 . If $G$ is a sporadic, alternating or symmetric group, then, for example, there does not exist a $\{2,5\}$-Hall subgroup of $G$ (for the symmetric groups see [9]). If $G=P S L(r, q)$, with $q=p^{f}$, then there does not exist a $\{p, t\}$-Hall subgroup for any prime $t$ such that $(t, q(q-1))=1$, except for $\operatorname{PSL}(3,2) \cong \operatorname{PSL}(2,7), \operatorname{PSL}(5,2)$ for which the statement holds with $t=7$, and $\operatorname{PSL}(3,3)$, where a $\pi_{1}$-Hall subgroup is in fact a $\{2,3\}$-Hall subgroup (see [23, Theorem 2.3.2]).

\section{Hall coverings}

In this section we want to prove the following:

THEOREM 4.1. Let $G$ be a group satisfying (*). Then $G$ admits a Hall covering if and only if $G / \operatorname{Fit}(G)$ is isomorphic to one of the following groups: $\operatorname{PSL}(2, q)$, $\operatorname{PSL}(3,4), \operatorname{PSL}(3, q)$ with $(3, q-1)=1, S z(q), A_{7}, M_{22}, M(q)$.

We begin with a lemma which allows us to reduce to the case of an almost simple group.

LEMMA 4.2. Let $G$ be a group satisfying (*). Then $G$ has a Hall covering if and only if $G / \mathrm{Fit}(G)$ has a Hall covering. 
Proof. This is Lemma 3.5 (ii).

We have proved in the preceding sections that if a group $G$ has a Hall cover, then $G$ has a $\pi_{1}$-Hall subgroup (see Corollary 3.5 ). It is therefore enough to examine the almost simple groups $G$ belonging to Table 1 .

Alternating groups Since $A_{5} \simeq \operatorname{PSL}(2,5)$, we suppose $r \geq 7$. Then the element (12) (34) $(5 \cdots r)$ of order $2(r-4)$ fixes no point and therefore it cannot be contained in a subgroup of $A_{r}$ isomorphic to $A_{r-1}$. Therefore $A_{r}(r \geq 7$ a prime) does not admit Hall coverings with $s=2$.

It is easy to see that $A_{7}$ admits a Hall covering with $t=s=3$.

Sporadic groups $M_{11}$ contains elements of order 6 but no subgroups of index 55 or 5 or 11 contains such elements.

$M_{22}$ does not contain subgroups of index $5 \cdot 7,5 \cdot 11$ or $5 \cdot 7 \cdot 11$ (see [5]). It can be easily seen that the $\{5,7\}^{\prime}$-Hall subgroups, together with the 5-Sylow and the 7-Sylow subgroups are a Hall covering of $M_{22}$ with $t=s=3$.

$M_{23}$ contains elements of order 15 , while none of its $\{2,3,5,7\}$-subgroups contain elements of order 15 . Therefore $M_{23}$ does not admit Hall coverings.

$J_{1}$ contains elements of order 15 but the only $\pi$-Hall subgroups with $\{3,5\} \subseteq \pi$, are isomorphic to $A_{5} \times C$ with $C$ a cyclic group of order 2 .

$\operatorname{PSL}(2, q) \quad$ It is well known that $\operatorname{PSL}(2, q)$ is a group with a partition and it admits a covering with $\pi_{i}$-Hall subgroups, for $i=1,2,3$ (see [12]). Moreover if $3<q \not \equiv 1(4)$, then the Borel subgroup of order $q(q-1) /(2, q-1)$ is a $\pi(q(q-1))$-Hall subgroup. Then, in this case, it also admits a partition with $\pi((q+1) /(2, q-1))$-Hall and $\pi(q(q-1))$-Hall subgroups. A subgroup containing a $p$-Sylow subgroup of $G$ must be contained in a Borel subgroup, then the only other possibility is to have a $\pi\left(q^{2}-1\right)$ Hall subgroup. We are then in the case of $G$ factorizable again and the only case we have to consider is $\operatorname{PSL}(2,11)$, with $A \cong A_{5}$ as a $\{2,3,5\}$-Hall subgroup. But there is an element of order 6 in $\operatorname{PSL}(2,11)$, which is not contained in any $\{2,3,5\}$-Hall subgroup.

$\operatorname{PSL}(3,4) \quad$ In this case every $\pi_{i}$ contains only a prime, and therefore there is a covering with the Sylow subgroups. We recall that $|G|=2^{6} \cdot 3^{2} \cdot 5 \cdot 7$. Moreover a 2-Sylow subgroup must be contained in a parabolic subgroup. By the remark at the beginning of the proof, there exists three conjugacy classes of parabolic subgroups: one of order $2^{6} \cdot 3$, which is not a $\{2,3\}$-Hall, and two of order $2^{6} \cdot 3 \cdot 5$. Moreover the only subgroups containing a Singer cycle are those of order 21 . Therefore the only possibility is a Hall covering with $\sigma_{1}=\pi_{1}=\{2\}$ and $\sigma_{2}=\pi_{4}=\{7\}$. If $H$ is a $\{3,5\}$-Hall subgroup, then $H$ should be contained in a maximal subgroup $M$ with $M \cong A_{6}$ (see [5]). But $A_{6}$ hasn't a $\{3,5\}$-Hall subgroup. 
$\operatorname{PSL}(r, q) \quad$ If $G=P S L(r, q)$, then $M=P_{1^{\prime}}$ or $M=P_{r^{\prime}}$ is a maximal parabolic subgroup, and also a $\pi_{1}$-Hall subgroup of $G$. Then $|M|=q^{r-1}(q-1)|S L(r-1, q)|$, since $(r, q-1)=1$. Then $M$ is a $\sigma_{1}$-Hall subgroup and $B=\left\langle x_{r}\right\rangle$ is a $\sigma_{2}=\pi_{2}$-Hall subgroup of $G$, where $x_{r}$ is a Singer cycle of order $\left(q^{r}-1\right) /(q-1)$. Moreover any $\sigma_{1}$-Hall subgroup is contained in a maximal subgroup and the only maximal subgroups with order divisible by $|G|_{\pi_{1}}$ are those isomorphic to $M$ (see [20]). It can be proved (see [3, Proposition 3.3]) that if $r \geq 5$, there exists an element $x$ in $P S L(r, q)$ of order

$$
b=\frac{q^{t}-1}{q-1} \frac{q^{r-t}-1}{q-1} .
$$

Moreover $b$ does not divide the following products

$$
\prod_{i=1, \ldots, s}\left(q^{j_{i}}-1\right) \quad \text { for } 1 \leq j_{i} \leq r-1, \sum_{i=1, \ldots, s} j_{i}=r-1
$$

and $b$ does not divide $q^{r}-1$. But then $x$ does not belong neither to a $\pi_{1}$-Hall subgroup nor to a $\pi_{2}$-Hall subgroup. Therefore, also in this case, $G$ cannot have a Hall covering.

If $r=3$, then there are two coverings: with the conjugates of a Singer cycle and with one of the two classes of maximal parabolic subgroups of $G$ :

$$
\mathscr{H}_{1}=\left\{P_{1^{\prime}}^{g},\left\langle x_{3}\right\rangle^{g} \mid g \in G\right\}, \quad \mathscr{H}_{2}=\left\{P_{r^{\prime}}^{g},\left\langle x_{3}\right\rangle^{g} \mid g \in G\right\} .
$$

This is proved in [3, Proposition 4.1 and Corollary 4.2].

$S z(q)$ By [12, Theorem 3.10, cap XI], the Suzuki groups admits a partition with $\pi_{i^{-}}$ Hall subgroups. Moreover, $G$ admits a $\pi_{1} \cup \pi_{2}$-Hall subgroup, which is a Frobenius group of order $q^{2}(q-1)$. Therefore, there are two kinds of coverings with Hall subgroups:

(i) $\pi_{1}, \pi_{2}, \pi_{3}, \pi_{4}$;

(ii) $\pi_{1} \cup \pi_{2}, \pi_{3}, \pi_{4}$.

Almost simple groups Let $G$ be an almost simple group which admits a Hall covering.

We recall that $\pi(G / S) \subseteq \pi(G)$, by [26, Theorem A (d)]. Therefore if $\mathscr{H}=$ $\left\{H_{1}, H_{2}, \ldots, H_{r}\right\}$ is a Hall covering of $G$, then $\mathscr{H}_{S}=\left\{H_{1} \cap S, H_{2} \cap S, \ldots, H_{r} \cap S\right\}$ is a Hall covering of $S$. We only have to consider the almost simple non simple groups, that is groups $G$ such that $S<G \leq \operatorname{Aut}(S)$, with $S$ a simple non-abelian group admitting a Hall covering.

If $G=S_{7}$, then $\pi_{1}(G)=\{2,3,5\}$ and the only subgroup of index 7 of $S_{7}$ is isomorphic to $S_{6}$. But $S_{6}$ does not contain elements of order 10 , as a $\{2,3,5\}$-Hall subgroup of $S_{7}$ should. 
$\operatorname{PSL}(2, q) \leq G \leq \operatorname{Aut}(\operatorname{PSL}(2, q))$ We first consider the case in which $G=$ $\operatorname{PSL}\left(2,2^{n}\right)\langle\alpha\rangle$ and $\alpha$ is a field automorphism of order 2. We recall that $C_{S}(\alpha)=$ $\operatorname{PSL}\left(2, q_{0}\right)$, where $q_{0}^{2}=q$, while $C_{B}(\alpha)=B_{0}$ of order $q_{0}\left(q_{0}-1\right)$. Therefore there exists an element $x \in C_{S}(\alpha)$ of order $\left(q_{0}+1\right)$ such that $x \cdot \alpha$ has order $2\left(q_{0}+1\right)$ and is not contained in $S$. This element is not contained in any of the conjugate of $\widetilde{B}$, since there is no element of such order in $\widetilde{B}$, with $\widetilde{B}$ the $\pi_{1}$-Hall subgroup of $G$ previously described.

If $G=M(q)$, then by the preceding Proposition, we have $\tilde{N}$, a $\pi_{1}$-Hall subgroup of $G$. We observe that any element of $G$ is contained in one of the $\pi_{i}$-Hall subgroups, and therefore we have the following covering:

$$
\left(\cup_{g} \widetilde{N}^{g}\right) \cup\left(\cup_{g} \widetilde{P}^{g}\right) \cup\left(\cup_{g} \widetilde{T}^{g}\right),
$$

$P$ is a $p$-Sylow subgroup of $G$, and $T$ is a (Singer) cycle of order $(q+1) / 2$.

$\operatorname{PSL}(3, q)\langle\alpha\rangle \quad$ By Proposition 3.1, there exists a $\pi_{1}$-Hall subgroup $\widetilde{P}_{1^{\prime}}$. But there exists an element of order $3(q-1)$ which is not contained in $\widetilde{P}_{1^{\prime}}$. The same is true if we consider the other class $P_{3^{\prime}}$ of $\pi_{1}$-Hall subgroups of $S$.

\section{Further remarks}

As already mentioned, the class of $C N$-groups is related to the groups admitting a Hall covering. It is not difficult to verify that if a group $G$ admits a nilpotent Hall covering (that is a Hall covering in which all the subgroups of the covering are nilpotent) then $G$ is a $C N$-group. It is also true that if $G$ is a $C N$-group, then $G$ admits a nilpotent Hall covering, using, for example, [7, Theorem 14.1.7].

We recall that the simple groups with a partition have been classified by Suzuki (see, for example, [22, Section 3.5]): they are $\operatorname{PSL}\left(2, p^{n}\right), p^{n}>3$ and $S z\left(2^{2 n+1}\right)$. They all admit a Hall covering, while the only simple $\mathrm{CN}$-group without a partition is $\operatorname{PSL}(3,4)$.

The soluble $C N$-groups are known (see [7, Theorem 14.1.5]), while Suzuki proved that a simple $C N$-group is isomorphic to one of the following list (see [12, Remark XI.3.12.a]):

(i) $\operatorname{PSL}\left(2,2^{n}\right)$ with $n>1$;

(ii) $\operatorname{PSL}(2, p)$ with $p$ Mersenne or Fermat prime;

(iii) $\operatorname{PSL}(2,9)$;

(iv) $\operatorname{PSL}(3,4)$;

(v) $S z\left(2^{2 n+1}\right)$ with $n>1$.

In the same paper [24, Theorem 4], Suzuki proved that a non-soluble $C N$-group is a CIT-group, that is a group of even order in which the centralizer of any involution is 
a 2-group. From the results of Higman [11], Suzuki [24] and Martineau [21], we also get:

THEOREM 5.1. Let $G$ be a non-soluble CN-group, then either

(1) $G$ is isomorphic to simple groups on Suzuki's list or

(2) $G$ is isomorphic to $M(9)$ or

(3) $G$ has a non trivial normal 2-subgroup $N$ and $G / N$ is isomorphic to $P S L\left(2,2^{n}\right)$ or to $\mathrm{Sz}\left(2^{2 n+1}\right)$. Moreover, $N$ is an elementary abelian group.

REMARK 2. The group $M(9)$ is a $C N$-group and it also admits a Sylow covering. This case was missing in the paper [2] on the non-soluble groups in which any element has order a power of a prime.

We note that we do not use character theory to prove Theorem 5.1, as it is done in [2]. We use a more elementary fact, which can be found in [11].

LEMMA 5.2 ([11, Theorem 8.1]). Let $H$ be a group with a normal 2-subgroup $T$ such that $H / T$ is dihedral of order 6. Let $h$ be an element of $H$ of order 3 acting fuxed point free on $T$, and let $R$ be a Sylow 2-subgroup of $H$. Then

(i) $T$ is of class at most 2 ;

(ii) if $|T|>4$, the class of $T$ is less than the class of any other subgroup of $R$ of index 2.

ProOF OF THEOREM 5.1. Let $G$ be a non-soluble $C N$-group, then $G$ has a nilpotent Hall covering. If $G$ is simple, then $G$ is in the Suzuki list. If $G$ is almost simple, then applying Theorem 4.1 we get that $G$ is isomorphic to $M(9)$ (see also [24, Theorem 3]).

By the above mentioned results of Suzuki, it is sufficient to prove the theorem for CIT-groups.

Let now $N$ be the maxima! normal soluble subgroup of $G$; then, if $\bar{G}=G / N$ we have $Z(\bar{G})=1$ and $O_{2^{\prime}}(\bar{G})=1$. We suppose $N \neq 1$ and, by Lemma 3.2 (i), we know that $N=\operatorname{Fit}(G)$. We first prove that $N$ is a 2-group. In fact $N$ is nilpotent and we can therefore assume that it is an $r$-group. If $r \neq 2$ then any Sylow 2-subgroup $\bar{S}$ of $\bar{G}=G / N$ acts fixed point free over $N$. Then $\bar{S}$ is a cyclic or a generalized quaternion group (see $[7,10.3 .1]$ ). In the first case $\bar{G}$ has a normal 2 -complement; in the second case by the Brauer-Suzuki Theorem (see [7, Chapter 12] and recall that $O_{2^{\prime}}(\bar{G})=1$ ) we get $Z(\bar{G}) \neq 1$. In both cases we get a contradiction. Therefore $N$ is a 2 -group.

Since $G$ is a $C I T$-group, any Sylow $2^{\prime}$-subgroup of $G$ acts fixed point free over $N$, and it is therefore cyclic. This implies that $\bar{G}$ is isomorphic to $\operatorname{PSL}\left(2,2^{n}\right), S z(q)$ or $\operatorname{PSL}(2, p)$ with $p$ a Fermat or Mersenne prime and $p>5$. If $\bar{G}$ is isomorphic to $\operatorname{PSL}(2, p)$ with $p$ Fermat or Mersenne prime and $p>5$, a Sylow 2-subgroup $\bar{S}$ of $\bar{G}$ is dihedral of order at least 8 . If $\bar{T}$ is an elementary abelian 2-subgroup of $\bar{G}$, 
then $|T|=4$ and $\bar{H}=N_{\bar{G}}(\bar{T})$ is isomorphic to $S_{4}$. We can apply lemma 5.2 to the preimages $H$ and $T$ of $\bar{H}$ and $\bar{T}$ in $G$ and $R$ a Sylow 2-subgroup of $H$. In particular $T$ has class 2 , otherwise $T \leq C_{G}(N) \leq N$.

Let $\bar{T}^{*}$ be an elementary abelian subgroup of order 4 of $\bar{H}$, distinct from $\bar{T}$. If $T^{*}$ is the preimage of $\bar{T}^{*}$ in $G$, then $T$ and $T^{*}$ are isomorphic. But $T^{*}$ is a subgroup of index 2 of $R$ and therefore, by Lemma 5.2, $T^{*}$ has class strictly less than the one of $T$.

The actions of $H=\operatorname{PSL}\left(2,2^{n}\right)=S L\left(2,2^{n}\right)$ or $H=S z\left(2^{2 n+1}\right)$ over an elementary abelian group $N$ are described respectively in [11, Theorem 8.2], and in the main theorem of [21]. The semidirect product $G=N H$ obtained by these actions is a CIT-group.

\section{References}

[1] Z. Arad and E. Fisman, 'On finite factorizable groups', J. Algebra 86 (1984), 522-548.

[2] R. Brandl, 'Finite groups all of whose elements are of prime power order', Boll. Un. Mat. Ital. A (5) 18 (1981), 491-493.

[3] D. Bubboloni and M. S. Lucido, 'Coverings of linear groups', Comm. Algebra 30 (2002), 21432159.

[4] R. W. Carter, Simple groups of Lie type (J. Wiley, London, 1972).

[5] J. Conway, R. Curtis, S. Norton, R. Parker and R. Wilson, Atlas of finite groups (Clarendon Press, Oxford, 1985).

[6] J. D. Dixon and B. Mortimer, Permutation groups (Springer, New York, 1996).

[7] D. Gorenstein, Finite groups (Harper \& Row, New York, 1968).

[8] F. Gross, 'Conjugacy of odd order Hall subgroups', Bull. London Math. Soc. 19 (1987), 311-319.

[9] P. Hall, 'Theorems like Sylow's', Proc. London Math. Soc. (3) 6 (1956), 286-304.

[10] G. Higman, 'Finite groups in which every element has prime power order', J. London Math. Soc. 32 (1957), 335-342.

[11] - Odd characterisations of finite simple groups, Lectures at The University of Michigan (Summer semester, 1968).

[12] B. Huppert and N. Blackburn, Finite groups III (Springer, Berlin, 1982).

[13] N. Iiyori and H. Yamaki, 'Prime graph components of the simple groups of Lie type over the field of even characteristic', J. Algebra 155 (1993), 335-343.

[14] A. S. Kondrat'ev, 'Prime graph components of finite simple groups', Mat. Sb. 180 (1989), 787-797: English translation: Math. of the USSR 67 (1990), 235-247.

[15] V. M. Levchuk and Ya. N. Nuzhin, 'The structure of Ree groups', Algebra i Logika 24 (1985), 26-41; English translation: Algebra and Logic 24 (1985), 16-26.

[16] M. W. Liebeck, 'On the orders of maximal subgroups of the finite classical groups', Proc. London Math. Soc. (3) 50 (1985), 426-446.

[17] M. W. Liebeck and J. Saxl, 'On the orders of maximal subgroups of the finite exceptional groups of Lie type', Proc. London Math. Soc. (3) 55 (1987), 299-330.

[18] M. S. Lucido, 'Prime graph components of finite almost simple groups', Rend. Sem. Mat. Univ. Padova 102 (1999), 1-22. ('Addendum ... ', Rend. Sem. Mat. Univ. Padova 107 (2002), 1-2.)

[19] G. Malle, 'The maximal subgroups of ${ }^{2} F_{4}\left(q^{2}\right)$ ', J. Algebra 139 (1991), 52-69.

[20] G. Malle, J. Saxl and T. Weigel, 'Generation of classical groups', Geom. Dedicata 49 (I994), $85-116$. 
[21] P. Martineau, 'On 2-modular representations of the Suzuki groups', Amer. J. Math. 94 (1972), 55-72.

[22] R. Schmidt, Subgroup lattices of groups (De Gruyter, Berlin, 1994).

[23] E. L. Spitznagel, Jr., 'Hall subgroups of certain families of finite groups', Math. Z. 97 (1967), 259-290.

[24] M. Suzuki, 'Finite groups with nilpotent centralizer', Trans. Amer. Math. Soc. 99 (1961), 425-470.

[25] — 'On a class of doubly transitive groups', Ann. of Math. (2) 75 (1962), 105-145.

[26] J. S. Williams, 'Prime graph components of finite groups', J. Algebra 69 (1981), 487-513.

[27] G. Zacher, 'Sull' ordine di un gruppo finito risolubile somma dei sottogruppi di sylow', Atti Acc. Naz. Lincei (8) 20 (1956), 171-174.

[28] _ _ 'Sui gruppi finiti somma dei loro sottogruppi di Sylow', Rend. Sem. Mat. Univ. Padova 27 (1957), 267-275.

Dipartimento di Matematica Applicata

e Informatica

Università "Ca' Foscari" di Venezia

Via Torino 155

31073 Venezia Mestre

Italy

e-mail: jabara@dsi.unive.it
Dipartimento di Matematica

e Informatica

Università di Udine

Via delle Scienze 208

I-33100 Udine

Italy

e-mail: mslucido@dimi.uniud.it 